Voix et Images

volxetimages

\title{
Mort et renaissances de l'écrivain maudit
}

Lectures de l'oeuvre et de la figure d'Hubert Aquin dans l'essai

québécois contemporain

Death and Rebirths of the écrivain maudit

Readings of the Work and Figure of Hubert Aquin in

Contemporary Quebec Essays

Muerte y renacimientos del escritor maldito

Lecturas de la obra y la figura de Hubert Aquin en el ensayo
quebequense contemporáneo

\section{Martine-Emmanuelle Lapointe}

Volume 38, numéro 1 (112), automne 2012

Relectures d'Hubert Aquin

URI : https://id.erudit.org/iderudit/1013446ar

DOI : https://doi.org/10.7202/1013446ar

Aller au sommaire du numéro

\section{Éditeur(s)}

Université du Québec à Montréal

ISSN

0318-9201 (imprimé)

1705-933X (numérique)

Découvrir la revue

Citer cet article

Lapointe, M.-E. (2012). Mort et renaissances de l'écrivain maudit : lectures de l'oeuvre et de la figure d'Hubert Aquin dans l'essai québécois contemporain. Voix et Images, 38(1), 27-41. https://doi.org/10.7202/1013446ar
Résumé de l'article

Le présent article s'attache à deux des modes de lecture de l'oeuvre aquinienne qui traversent la critique et l'essai québécois contemporains. La lecture biographique, plus volontiers mythifiante, de certains essayistes et biographes aquiniens permettra, d'une part, de réfléchir à la fascination qu'exerce encore aujourd'hui le sacrifice d'Aquin sur ses lecteurs contemporains. D'autre part, il s'agira de mieux cerner l'inscription de l'oeuvre aquinienne dans les dossiers que la revue Liberté a fait paraître entre 2006 et 2012. En réactualisant la dimension politique de l'oeuvre d'Aquin, les auteurs de la revue réfléchissent à la fois sur la quasi-disparition de l'engagement politique dans la sphère culturelle et sur le décentrement de la parole littéraire à l'époque contemporaine. D'un point de vue comme de l'autre, Aquin et son oeuvre méritent d'être relus, d'être tirés de la bibliothèque ou du tombeau afin de répondre, ne serait-ce que par la négative, aux angoisses et aux obsessions des contemporains. Nous entendons en somme dépasser certaines idées reçues sur la construction des figures auctoriales, lesquelles viseraient selon certains auteurs à "simplifier [l'oeuvre] pour [la] rendre comestible ». Malgré ses limites évidentes, le mythe offre à l'oeuvre une possible survie dans le concert des médias contemporains et permet de saisir les enjeux d'une certaine représentation, historique sans doute, de la littérature québécoise. 


\section{MORT ET RENAISSANCES DE L'ÉCRIVAIN MAUDIT}

Lectures de l'œuvre et de la figure d'Hubert Aquin dans l'essai québécois contemporain* 1

$+++$

MARTINE-EMMANUELLE LAPOINTE

Université de Montréal

Il semble bien que [...] l'écrivain maudit serait une sorte de mythe qui, par l'impact de la mise en marché et de la diffusion, serait en train de devenir périmé... ou quelque chose d'approchant: quelque chose de rétrograde... Je me suis permis de déduire que [...] l'écrivain maudit se devait - pour être à la mode du

jour — de mourir au plus vite - faute de quoi il sera frappé d'interdit... voire jeté à la poubelle ${ }^{2} \ldots$

À première vue, tout semble éloigner l'œuvre et la figure d'Hubert Aquin de l'ethos contemporain : l'engagement politique, la radicalité des thèses défendues dans certains essais, l'exploration formelle, l'extrême raffinement de la langue confinant parfois à l'hermétisme, traits si souvent relevés par les critiques aquiniens, seraient quasi absents des œuvres littéraires publiées au Québec depuis les années 1980. Ces dernières, du moins selon les commentateurs les plus intransigeants, se seraient attiédies, seraient «écrites dans une langue improbable [...] éman[ant] de nulle part ${ }^{3}$ ", témoins d'une forme de "normalisation d'une littérature » jadis " petite, unifiée et moderne ${ }^{4}$ ", d'un décentrement ${ }^{5}$ de la culture qui confirmerait l'inévitable disjonction des discours politique et littéraire. C'est sans doute Olivier Kemeid qui a le mieux décrit le malentendu qui semble détourner Aquin de ses lecteurs contemporains dans son article « Le refus de la modernité » consacré au fameux «Combat des livres » de Radio-Canada qui, en 2006, avait réservé un sort funeste à Prochain épisode:

La littérature des détracteurs de Prochain épisode, c'est celle des cloîtrés, des nécessiteux de l'ornière, des plie-genoux. C'est la littérature utilitaire, mièvre, si populaire

$$
+++
$$

* Pour obtenir la liste des sigles utilisés dans cet article et les références complètes aux œuvres d'Hubert Aquin, voir p. 11-12. 1 Les recherches qui ont conduit à la rédaction de cet article s'inscrivent dans le cadre du projet «Tombeaux de la littérature québécoise contemporaine dans l'essai et la critique (1980-2010)» subventionné par le Fonds de recherche du Québec - société et culture (FQRSC). 2 Hubert Aquin, "La mort de l'écrivain maudit » (M1, 201). 3 Pierre Lefebvre, «L'âge de l'institution", Liberté, vol. L, nº 4, décembre 2009, p. 9.4 François Ricard, «Remarques sur la normalisation d'une littérature», Écriture, nº 31, automne 1988, p. 11.5 Voir notamment le chapitre «Le décentrement de la littérature (depuis 1980)», Michel Biron, François Dumont et Élisabeth Nardout-Lafarge, avec la collaboration de Martine-Emmanuelle Lapointe, Histoire de la littérature québécoise, Montréal, Boréal, coll. «Boréal compact», 2010, p. 531-626. 
et compréhensive qu'elle en embrigade les cerveaux mous : c'est la littérature "près de nous ", qui nous parle, qui nous ressemble tellement. Une fois de plus au Québec, la mimesis est au pouvoir. Ce qui importe, c'est la retranscription fidèle du réel, afin que nul filtre ne vienne altérer notre conception du monde. Ce qui importe, c'est de rester entre nous, chez nous. L'imaginaire est alors relégué aux oubliettes de l'Histoire. Le dérangeant, le pas beau pas propre, l'ardu, l'œuvre ouverte au sens où l'entend Umberto Eco, c'est-à-dire l'œuvre aux ramifications multiples, aux interprétations plurielles, l'œuvre qui exige, faut-il la nommer, une lecture - et non un assemblage visuel de signes qui, lui, permet de reconnaître ce qu'il y a dans nos céréales - bref tout ce qui demande un effort, serait réservé à une élite ${ }^{6}$.

Or le mépris manifesté à l'égard de Prochain épisode par les lecteurs invités au «Combat des livres» est justement ce qui confirme, selon Kemeid, la survie, l'irrévérence, le caractère toujours explosif d'une œuvre qui arrive encore à choquer les bienpensants. Dans la suite de son article, Kemeid va jusqu'à affirmer que «jamais Prochain épisode ne [lui] a paru aussi vivant, actuel, brûlant ${ }^{7}$ ». Cette lecture - engagée, pour le moins - que propose Olivier Kemeid montre bien que, malgré le malentendu et peut-être grâce à lui, la figure et l'œuvre d'Aquin jouent toujours un rôle signifiant dans plusieurs des récits qui accompagnent la littérature québécoise contemporaine. Elles permettraient même, par-delà le temps et les mouvances idéologiques, de rétablir le lien entre la période contemporaine et l'héritage littéraire des années 1960-1970.

La réception de l'œuvre d'Hubert Aquin diffère à plusieurs égards de celles des autres écrivains majeurs des années 1960-1970 - Jacques Ferron, Gaston Miron, Marie-Claire Blais, Réjean Ducharme, pour ne nommer que ceux-là - en ce qu'elle s'inspire de manière souvent indifférenciée de l'œuvre et de la biographie, si ce n'est de la thanatographie de l'auteur. Au cours des dix dernières années, critiques, écrivains et biographes ont souvent mis l'accent sur la relation affective et existentielle qu'ils ont en quelque sorte nouée avec l'œuvre d'Aquin. Loin de constituer un héritage moribond, cette dernière offrirait une lecture du monde, une philosophie de l'existence, voire une éthique, qui seraient toujours d'actualité. Le suicide de l'auteur apparaîtrait même aux yeux de certains comme une forme de résistance, certes radicale, à la morosité culturelle contemporaine.

Peut-on pour autant en conclure à l'existence d'un mythe d'Aquin ? La question est piégée, et mérite d'être traitée avec circonspection. Dans Hubert Aquin blues, Richard Dubois s'en prend aux lectures qui ont conduit à la création d'un mythe chez les «commentateurs, surtout les journalistes littéraires » qui ont simplifié l'œuvre pour la «rendre comestible ${ }^{8}$ ». Il réduit ainsi le processus de mythification à une " accumulation de témoignages" et à «la louange de tout et de son contraire ${ }^{9}$ ». La louange, le couronnement du génie, la célébration de l'œuvre participent assurément à la fabrication du mythe, mais sont peu de chose au regard de l'homologie entre

6 Olivier Kemeid, «Le refus de la modernité», Liberté, vol. XLVIII, n 2, mai 2006, p. 84.77 Ibid., p. 86.

8 Richard Dubois, Hubert Aquin blues, Montréal, Boréal, 2003, p. 40. 9 Ibid., p. 44-45. Dubois souligne. 
certains des thèmes de l'œuvre aquinienne - l'échec, le suicide, l'idéal révolutionnaire, entre autres - et la biographie de l'écrivain. Certes douteux, cet amalgame de références est aussi parfois ce qui permet à l'œuvre, et à la littérature qu'elle représente, de se doter de traditions de lecture internes. Malgré ses limites évidentes, le mythe offre à l'œuvre une possible survie dans le concert des médias contemporains et permet de saisir les enjeux d'une certaine représentation, historique sans doute, de la littérature québécoise. Comme le note Jean-Luc Nancy, «le mythe [est] le plus souvent celui d'un héros isolé. À un titre ou à un autre, ce héros fait communier la communauté - et il la fait communier toujours, en définitive, dans la communication, opérée en lui-même, de l'existence et du sens, de l'individu et du peuple ${ }^{10}$ ». Sujet-Nation à sa manière, «mosaïque discursive s'imposant au fil du temps et variant en fonction des vecteurs interdiscursifs qui le traversent ${ }^{11}$ ", pour reprendre les mots de Pascal Brissette, Aquin - sa figure, son œuvre - n'est pas un mythe au sens strict, mais il s'accompagne de récits mémoriels, de lectures diverses, de motifs récurrents qui lui confèrent un rôle, sans conteste important, dans l'histoire de la littérature québécoise.

Sans prétendre à l'exhaustivité ${ }^{12}$, le présent article s'attache à deux des modes de lecture de l'œuvre aquinienne qui traversent la critique et l'essai québécois contemporains. La lecture biographique, plus volontiers mythifiante, de certains essayistes et biographes aquiniens permettra, d'une part, de réfléchir à la fascination qu'exerce encore aujourd'hui le sacrifice d'Aquin sur ses lecteurs. D'autre part, il s'agira de mieux cerner l'inscription de l'œuvre et de la figure aquiniennes dans les dossiers que la revue Liberté a fait paraître entre 2006 et 2012. Les auteurs de la revue privilégient les textes essayistiques et les interventions publiques d'Aquin. En réactualisant ainsi la dimension politique de l'œuvre de l'auteur, ils réfléchissent à la fois à la quasidisparition de l'engagement politique dans la sphère culturelle et au décentrement de la parole littéraire. D'un point de vue comme de l'autre, Aquin et son œuvre méritent d'être relus, d'être tirés de la bibliothèque ou du tombeau afin de répondre, ne seraitce que par la négative, aux angoisses et aux obsessions des contemporains.

\section{LE SPECTRE AU CRÂNE FRACASSÉ}

Plusieurs des essais consacrés à l'œuvre d'Aquin depuis 1997 renoncent à l'appareil critique et aux cadres conceptuels des ouvrages savants pour renouer avec une conception quasi existentielle de la littérature. Oscillant entre l'essai, la confession et

$$
++
$$

10 Jean-Luc Nancy, «Le mythe interrompu», La communauté désœuvrée, Paris, Christian Bourgois, coll. «Détroits», 2004, p. 129. Dans son roman Ça va aller, Catherine Mavrikakis revisite le sujet de la communion avec la communauté opérée par le mythe d'Aquin: "Je pense à celui qui a inventé la mort, mais qui ne nous a pas permis d'inventer la vie. Je pense à Aquin. Je dévore le corps de ses œuvres, je communie dans la défaite.» (Catherine Mavrikakis, Ça va aller, Montréal, Leméac, 2002, p. 46) 11 Pascal Brissette, Nelligan dans tous ses états. Un mythe national, Montréal, Fides, coll. «Nouvelles études québécoises», 1998, p. 30.12 Les ouvrages et les articles qui ont servi à l'élaboration de cet article sont recensés dans la bibliographie d’Hubert Aquin (19972011) accompagnant le présent dossier. 
la fiction, les essais Hubert Aquin. La course contre la vie ${ }^{13}$ et La fatigue d'être ${ }^{14}$ de Jacques Beaudry, parus respectivement en 2006 et $2008^{15}$, s'attachent aux thèmes du mal-être, du sacrifice de soi et de l'opprobre social qui auraient conduit nombre d'écrivains au suicide. Au sein de ce répertoire, Hubert Aquin occupe bien sûr une place centrale: personnage principal de Hubert Aquin. La course contre la vie, il est accompagné dans La fatigue d'être de deux autres "suicidés de la société», Saint-Denys Garneau et Claude Gauvreau. Les ouvrages Hubert Aquin blues de Richard Dubois et Renaissances. Vivre avec Joyce, Aquin, Yourcenar ${ }^{16}$ de Guylaine Massoutre tentent eux aussi, chacun à sa manière, de se détacher des analyses littéraires plus classiques. Chez Dubois, il s'agit pour l'essentiel d'adopter l'attitude du franc-tireur afin de rompre avec une conception trop élitiste de la littérature, laquelle ne peut «se contenter d'être fluflutée comme une tisane par les honorables fatigués d'une Académie quelconque ${ }^{17}$ ». Massoutre, quant à elle, accorde à son ouvrage le statut de fiction, selon l'appellation générique figurant sur la page couverture. Elle entrelace le parcours de son héroïne Christine Forestier - d'après le nom d'un des personnages de L'antiphonaire - avec celui d'Aquin, établissant des correspondances entre leurs expériences existentielles et littéraires. Chez les trois auteurs, il s'agit bien de vivre avec Aquin, pour reprendre le sous-titre de l'ouvrage de Guylaine Massoutre. L'œuvre et la figure de l'auteur sont réinvesties de manière affective, réactualisées afin de servir le présent. Pour Richard Dubois, «le "mort" bouge encore, ses livres ont aujourd'hui du sens, et ils peuvent aider à mieux comprendre, à mieux s'insérer dans le monde du World Trade Center effondré ${ }^{18}$ ». Pour Jacques Beaudry, sans doute le plus radical des trois auteurs, l'écrivain apparaît comme le miroir d'angoisses collectives qu'il vit intensément, dans une sorte de dépense insensée d'énergie vitale. Vidé, exsangue, n'arrivant pas à transcender son mal-être dans l'écriture, il est condamné au «tombereau [du] suicidé» (FE, 122). La fiction de Guylaine Massoutre, quant à elle, abolit littéralement la frontière entre œuvre et biographie. Hubert Aquin apparaît comme un compagnon de lecture, de voyage et de découvertes, voire une sorte de baromètre des expériences collectives de la Révolution tranquille. Dans les trois ouvrages, le procès d'identification du lecteur avec l'œuvre est loin d'être dénoncé. Il est plutôt mis en valeur, comme s'il permettait

$$
++
$$

13 Jacques Beaudry, Hubert Aquin. La course contre la vie, Montréal, Hurtubise HMH, coll. «Constantes », 2006, 124 p. Désormais, les références à cet ouvrage seront indiquées par le sigle HAC suivi du folio, et placées entre parenthèses dans le texte. 14 Jacques Beaudry, La fatigue d'être. Saint-Denys Garneau, Claude Gauvreau, Hubert Aquin, Montréal, Hurtubise HMH, coll. «Constantes», 2008, 140 p. Désormais, les références à cet ouvrage seront indiquées par le sigle FE suivi du folio, et placées entre parenthèses dans le texte. 15 Les deux ouvrages comportent des appendices justifiant l'absence dans le corps du texte de références précises aux œuvres citées: «Il ne convenait pas de surcharger le texte de renvois et de références. Le lecteur attentif saura bien se repérer: avec les indications de dates, se reporter aux journaux des écrivains et à leur correspondance; avec les mentions de titres et les noms de personnes, retrouver les textes ou dans ceux-ci les passages sur un personnage en particulier, en se servant des index des différentes éditions des écrits de Garneau, Gauvreau et Aquin » (FE, 123). Ce bref passage dessine le portrait du lecteur recherché par Jacques Beaudry: celui-ci doit se montrer attentif aux nuances du texte, familier des trois auteurs étudiés et assez hardi pour retourner aux sources des œuvres citées. 16 Guylaine Massoutre, Renaissances. Vivre avec Joyce, Aquin, Yourcenar, Montréal, Fides, 2007, 440 p. 17 Richard Dubois, Hubert Aquin blues, p. 11. 18 Ibid., p. 15. 
de revenir au fondement même de l'expérience littéraire. Sans toujours renoncer à la distance critique, chacun se permet de fabriquer son Aquin. Ce dernier sera essayiste plus que romancier chez Dubois, victime sacrifiée par une société étroite chez Beaudry, confident et objet de désir chez Massoutre.

Dans une telle perspective, le suicide d'Aquin n'est guère un sujet tabou. Au contraire, il équivaut chez certains auteurs non pas à une fin brutale ou à une rupture, mais à une sorte d'achèvement, de prolongement de l'œuvre écrite. Signant certes la disparition physique de l'écrivain, le suicide est également son ultime cri de libération ${ }^{19}$. Difficile de ne pas entendre, dans cette lecture du suicide, nombre de motifs, d'idées et de métaphores chers à Aquin. Dans plusieurs des textes aquiniens en effet, l'être porte en lui une lucidité dévorante, si aiguë qu'elle pousse souvent au suicide. Loin d'être infamant, ce dernier est indissociable du sens de l'honneur, voire de l'intégrité de celui qui se sait promis à un avenir médiocre et qui préfère la mort à une vie faite de compromissions et de ratages. Dans L'invention de la mort, René Lallemant prétend retrouver «le don de voir» (IM, 143) au moment où il prépare son suicide. «L'art de la défaite» file la comparaison entre le suicide et l'art militaire des patriotes. Dès leur première bataille en 1837, ces derniers auraient été «[c]onditionnés à la défaite comme d'autres le sont au suicide parce qu'ils ont de l'honneur» (M2, 135). Dans Prochain épisode, Aquin évoque «le revolver avec lequel Papineau aurait mieux fait de se suicider» $(P E, 155)$ et dans Obombre, son ultime roman (inachevé), il écrit: «Non ce n'est pas la vie qui console de la mort, mais bien la mort de la vie» (M1, 339). Mieux vaut mourir que de mal vivre, que de mal s'engager ou que de mal écrire, semble affirmer Aquin au fil de ses œuvres.

Aussi douteuse soit-elle, l'homologie souvent établie entre les suicides fictifs et la mort volontaire d'Aquin constitue la source d'inspiration de différents ouvrages critiques et biographiques. L'on songera bien sûr à HA! A Self-Murder Mystery publié par Gordon Sheppard en 2003 qui, dès le chapitre I, présente une reconstitution des événements entourant le suicide d'Aquin dans le jardin de Villa Maria en mars $1977^{20}$. Si la perspective de Jacques Beaudry diffère à plusieurs égards de celle de Sheppard - qui, par souci de vraisemblance, va jusqu'à reproduire la lettre d'adieu laissée par Aquin à sa compagne Andrée Yanacopoulo -, elle n'en demeure pas moins fondée sur une fascination pour le sacrifice, voire le "self-murder» de l'artiste maudit. Dans Renaissances de Guylaine Massoutre, le personnage de Christine ne retient d'abord de la scène du suicide que sa nature mortifère : «son acte me faisait toucher la haine, le dégoût mortel de moi. [...] Mortifiée, j'ai rangé brusquement les bannières d'un art de vivre qui $n^{\prime}$ en avaient que l'apparence ${ }^{21} »$. Plus tard, elle accueille les «nouveaux émois» engendrés par «l'être perdu ${ }^{22}$ » et recompose un panthéon d'auteurs

19 Dans Hubert Aquin. La course contre la vie, Jacques Beaudry consacre quelques pages au lien entre suicide et cri de libération. Il y affirme notamment que « [l] cri permet à Aquin d'échapper à l'asile des mots où l'on tente de nous enfermer collectivement. L'énorme mécanisme de la rhétorique transporte l'être hors du monde, dans nos cerveaux. En pulvérisant le sien, Aquin replace l'être dans la vie: son acte est l'acte d'un vivant» (HAC, 25). 20 Gordon Sheppard, HA! A Self-Murder Mystery, Montréal/Kingston, McGill-Queen's University Press, 2003, 850 p. 21 Guylaine Massoutre, Renaissances, p. 174. 22 Ibid., p. 182. 
suicidés, parmi lesquels se trouve notamment Claude Gauvreau. Dans les essais de Jacques Beaudry, Aquin est littéralement transfiguré. Devenu personnage de fiction, il se présente comme une figure mythique, un "Christ anarchique ( $H A C, 34)$ semblable à Edipe, à Ulysse, à Hamlet et à Faust. Beaudry confère par là même à la mort d'Aquin une dimension symbolique et politique. Revisitant les grands thèmes de «Van Gogh, le suicidé de la société ${ }^{23}$ » d'Antonin Artaud, il oppose la marginalité de l'être unique, sa démesure quasi géniale, aux lois aveugles de la collectivité: «Aquin est le suicidé d'une société qui le réduit à rien, mais en même temps il est celui qui en se supprimant s'affirme totalement: le rien qui est zéro se voit comblé par un néant qui est plénitude et qui l'annule» (HAC, 27). Telle la révolution, souvent comparée au cri chez Aquin, le suicide apparaît chez plusieurs auteurs comme un acte libérateur, mais aussi comme une manière de sortir du temps et de l'histoire communautaires. En se suicidant, Aquin se soustrait aux manèges de sa société, il se choisit, il affirme sa souveraineté.

La figure d'écrivain ainsi esquissée se constitue à même une rhétorique du renversement des valeurs établies : la fatigue, la dépression et le suicide sont considérés comme des armes, deviennent les emblèmes d'une résistance totale ${ }^{24}$. Or, loin d'être inédite, cette rhétorique emprunte largement au répertoire des mythes et des valeurs chrétiens, conjuguant la rédemption par la souffrance au sacrifice de soi et à la sacralisation de la parole. De nombreuses références à la figure du Messie notamment, souvent tirées des textes mêmes d'Aquin, traversent l'argumentaire de Beaudry. C'est donc dans le droit fil d'une tradition ancienne que s'inscrivent les analyses littéraires et biographiques de l'essayiste. Comme le rappelle Pascal Brissette,

\begin{abstract}
[le mythe du pouvoir bienfaisant de la souffrance] ne peut guère être séparé d'avec le mythe chrétien qui le précède et l'excède, puisqu'il s'y greffe et apparaît comme une recomposition symbolique laïque, adaptée à la collectivité lettrée, du mythe chrétien. Il en a retenu non seulement tout un vocabulaire (l'assemblée littéraire comme «cénacle», etc.) et certaines images ou rites, mais une logique «à bascule» où le malheur est donné pour un signe d'élection et d'élévation, ainsi qu'un arsenal d'arguments, tel celui du «sacrifice», qui consiste à dire que la valeur d'une cause ou d'une œuvre donnée est fonction de la grandeur du sacrifice que l'auteur est prêt à consentir pour elle ${ }^{25} \ldots$
\end{abstract}

Fidèle à cette «logique à bascule», Beaudry n’hésitera pas à conférer à Aquin le titre de génie, mot surchargé s'il en est. L'essayiste renoue ainsi avec l'étymologie de "génie» qui, jusqu'à la fin du Xvire siècle, se conjuguait aux " "divine ardeur", "fureur démoniaque", "sublime folie", "inspiration surhumaine" 26 " des êtres exceptionnels.

$+++$

23 Antonin Artaud, Van Gogh, le suicidé de la société, Paris, Gallimard, coll. «L'imaginaire», 2001 [1947], 93 p. 24 Ce terme n'est pas anodin. Il apparait dans les titres des trois sections de l'ouvrage Hubert Aquin. La course contre la vie, soit «La guerre totale», «Le roman total» et «L'homme total». 25 Pascal Brissette, «Poète malheureux, poète maudit, malédiction littéraire. Hypothèses de recherche sur les origines d'un mythe», COnTEXTE, en ligne: http://contextes.revues.org/index1392.html (page consultée le 21 juin 2012). 26 Baldine Saint Girons, «Génie», Encyclopædia Universalis, en ligne: http://www.universalis.fr/encyclopedie/genie/(page consultée le 21 juin 2012). 
Une figure particulière, employée de manière récurrente dans les deux essais de Beaudry, mais aussi présente chez Gordon Sheppard et Guylaine Massoutre 27, pourrait bien condenser les thèmes associés à la génialité d'Hubert Aquin. Sorte de synecdoque morbide, elle consiste à représenter le suicide d'Aquin - et par là les thèmes du sacrifice de soi, de l'incompréhension de l'être génial et du chef-d'œuvre thanatographique - en évoquant le "crâne éclaté» $(F E, 14)$ de l'auteur suicidé. Leitmotiv des deux essais de Beaudry, la figure du crâne éclaté ou fracassé est littéralement présentée sous la forme d'une idée directrice dans Hubert Aquin. La course contre la vie. L'ouvrage s'ouvre, en effet, sur un exergue de Claude Gauvreau se lisant comme suit: «Recueillez les languettes de notre cervelle qui pendent au faîte des sapins; et après l'accomplissement de ce devoir, nous ferons tous ensemble un bon gueuleton de cervelle de veau. » (HAC, 11) Ici s'affirme, une fois de plus, le thème du sacrifice de soi, mais surtout l'opposition entre un «nous» et un "vous», aux frontières pourtant poreuses chez Gauvreau, sur laquelle Beaudry ne cessera d'insister. Dans le dernier chapitre, éloquemment intitulé "Le surhomme», Beaudry déclare, à la suite de Johann Georg Hamann, que «le génie est une couronne d'épines» (FE, 100101). Ce chapitre de trois pages entrelace les théories de Nietzsche, les métaphores messianiques, les allusions aux figures d'Hypérion, de Faust et d'Hamlet, les références aux œuvres de Miron, de Zweig, de Goethe, de Schelling, de Mann, de Holbein, de Dürer, de Valéry, de Teilhard de Chardin, et témoigne de la pratique analogique de Beaudry qui, plutôt que d'approfondir l'analyse des intertextes, privilégie l'accumulation, la superposition et le collage des fragments et des citations, laissant ainsi à son lecteur la liberté de décrypter ou non ses tissages discursifs.

Au sein de cet ultime chapitre, la figure du crâne "couronné d'épines», puis «fracassé» devient en quelque sorte la clé de lecture de l'ensemble de l'essai. C'est d'abord à l'extase mystique que renvoie la « couronne d'épines ». L'état mystique provoque «le dépassement de la raison» $(F E, 100)$ et annonce le fatal destin de Nietzsche et d'Aquin, génies tous deux foudroyés. Un peu plus loin apparaît le crâne des Ambassadeurs de Holbein, « un tableau obscur dont jaillit, pour Aquin, ce même éclair qui fit saisir à Nietzsche devant Le chevalier avec la Mort et le Diable de Dürer que "comprendre est une fin"» (FE, 102). Puis, sous l'effet d'un nouveau glissement sémantique, affleure le crâne d'Hamlet, «ramassé » par Faust dans un texte peu connu de Valéry. Enfin, les dernières lignes de l'essai ramènent au suicide historique d'Aquin :

quelques mots que le cadavre au crâne troué qui gît décervelé dans une allée des jardins de Villa Maria aurait, s'il s'était réincarné le jour d'après en lui-même vivant, sans doute prononcés: «Comment renouer une vieille relation humaine trop humaine puisque le crime de la veille m'a hissé au-delà du bien et du mal aimer - pareil, en cela, au promeneur, au promeneur inlassable de Sils Maria! J'ai franchi le seuil des sentiments humains et de la faiblesse. » (FE, 102)

$$
+++
$$

27 Voir notamment Guylaine Massoutre, Renaissances, p. 181. 
Grâce à ce dénouement, Jacques Beaudry respecte scrupuleusement la cohérence de sa pratique et de son propos. Empruntées à Trou de mémoire, les dernières lignes de son essai ramènent à l'épigraphe de Gauvreau, rejouent la scène épiphanique du suicide en insistant derechef sur sa dimension messianique, mais surtout réaffirment, par l'entremise des références à l'œuvre et à la biographie de Nietzsche, l'indissociabilité de la réalité historique et de la fiction. La valeur épiphanique du suicide est d'ailleurs intensifiée par l'allusion à Sils Maria, lieu où Nietzsche aurait eu la révélation de la loi de l'éternel retour, à l'origine de son ouvrage Ainsi parlait Zarathoustra. Siège de l'intellect et du génie, mais aussi symbole par excellence de la finitude humaine, la figure du crâne "troué», "décervelé», y signale bien plus qu'une curiosité morbide ; elle dit aussi l'importance de la dépense physique et du sacrifice corporel, vécus à même la chair de l'être. Rien d'étonnant à ce que le «crâne éclaté» figure à la première page de La fatigue d'être qui, bien que consacré à trois auteurs distincts, n'en reconduit pas moins le mythe de l'écrivain maudit.

Certes, l'on pourra arguer que les essais de Jacques Beaudry demeurent des exceptions dans le milieu littéraire québécois. Si le style, la rhétorique et l'érudition de l'auteur de Hubert Aquin. La course contre la vie et de La fatigue d'être s'avèrent singuliers, leur propos, entendu au sens large, circule pourtant dans de nombreux textes, des hommages publiés dans les journaux ${ }^{28}$ aux biographies d'Aquin ${ }^{29}$, en passant par les essais et les fictions mettant l'écrivain ou ses doubles en scène ${ }^{30}$. Sous la plume d'un Yvon Rivard, Hubert Aquin témoigne, au même titre que Saint-Denys Garneau, Gaston Miron, Jacques Brault et Pierre Vadeboncœur, de la survivance des héritages de la pauvreté propres à une certaine tradition québécoise. Comme dans Le milieu du jour qui prêtait à Hubert Aquin les traits de l'écrivain Nicolas, le suicide est pour Rivard un symptôme, voire un motif littéraire, plus qu'un fait «sociopsychologique»:

Si on veut bien oublier un instant que l'auteur des lignes précédentes s'est suicidé (et qui dit qu'un suicide invalide l'œuvre et la vie de son auteur?), si l'on veut bien cesser de lire les termes d'échec ou de pauvreté à la seule lumière de la sociopsychologie, ce texte d'Aquin fonde une pratique de l'échec qui conduit au succès, il postule

$$
+++
$$

28 Voir notamment le cahier «Livres» du journal Le Devoir soulignant le trentième anniversaire de la mort d'Aquin. Les premières lignes de ce cahier, signées Jacques Allard, synthétisent les différents aspects du mythe d'Aquin explorés par Jacques Beaudry dans ses essais : «Le 15 mars 1977, la date vous rappelle quelque chose? Il y a trente ans, ce jour-là, vers 14 h, Hubert Aquin a pris son fusil et s'en est allé pour toujours. Il s'était tant cherché qu'il a fini par se trouver. La violence l'habitait. Il l'a transformée en œuvre d'art pendant une trentaine d'années avant de la retourner contre lui-même, quand il n'a pas eu la force de continuer.» Jacques Allard, «Le jour où Hubert Aquin s'en alla", Le Devoir, 10 et 11 mars 2007, p. F1. 29 Voir, en plus de HA! A Self-Murder Mystery de Gordon Sheppard, Gordon Sheppard et Andrée Yanacopoulo, Signé Hubert Aquin. Enquête sur le suicide d'un écrivain, Montréal, Bibliothèque québécoise, 2002 [1985], 486 p.; Françoise Maccabée-Iqbal, Desafinado. Otobiographie de Hubert Aquin, Montréal, VLB éditeur, 1987, 461 p. 30 Voir notamment René Gingras, Le facteur réalité, Montréal, Leméac, coll. "Thêâtre/Leméac», 1985, 158 p.; Andrée Ferretti, Renaissance en Paganie, Montréal, l'Hexagone, coll. «Fictions», 1987, 81 p. ; Yvon Rivard, Le milieu du jour, Montréal, Boréal, 1995, 327 p. ; et Catherine Mavrikakis, Ça va aller. 
la possibilité d'un «art de la défaite» qui procède non pas uniquement de la peur de réussir mais d'un dépassement même des notions d'échec et de réussite ${ }^{31}$.

L'héritage de la pauvreté n'est pas que misérabilisme et impuissance; au contraire, il enseigne l'humilité et le dépouillement, incite à revoir les catégories communément admises du bien et du mal, de l'échec et de la réussite, de la pauvreté et de la richesse. Rivard ne procède pas à un simple renversement des valeurs établies, mais tend plutôt à invalider les binarismes conduisant immanquablement au triomphe d'une quelconque positivité.

\title{
LES LECTEURS «ASSOIFFÉS DE SENS »DE LA REVUE LIBERTÉ
}

Sous l'impulsion de son nouveau comité de rédaction ${ }^{32}$, la revue Liberté fait paraître en 2006 un dossier bilan consacré à la culture québécoise contemporaine, dont le titre, «La résistance culturelle», trahit clairement les intentions. Le texte liminaire de ce dossier, présenté comme un éditorial, constitue plutôt une sorte de texte programmatique voué à consolider les nouvelles vues du comité. Intitulé "Assoiffés de sens», il se revendique d'une filiation directe avec les fondateurs de la revue et l'esprit de la Révolution tranquille:

\begin{abstract}
Le mot d'ordre au moment de la fondation de la revue, en l'an de grâce 1959, était de «tenir compte, d'étape en étape, de l'évolution de la pensée, de la création sous toutes ses formes, de la vie artistique à travers toutes ses manifestations». Le but des fondateurs était, somme toute, assez simple: dresser l'état des lieux. Ce côté «inventaire», pressenti avec urgence, permettait une assise à tous ceux qui ressentaient le besoin de se réunir autour d'un lieu où la parole avait un poids et des dents. La Révolution tranquille s'éveillait, la résistance culturelle s'organisait et se donnait des armes: Liberté était l'une d'elles. Au cours des années 1960-1970, alors que l'on passait du Canada français au Québec, le projet de la revue gardait son sens : jamais l'agora qu'elle représentait n'avait connu une aussi forte ébullition ${ }^{33}$.
\end{abstract}

Vers la fin de l'article, le comité ouvre ses pages aux assoiffés de sens contemporains qui, faute d'un ancrage communautaire, sont vus comme des orphelins. Liberté devient littéralement leur future maison. À ce geste d'accueil se greffe le retour aux métaphores de la filiation, de l'appartenance, mais aussi de l'aménagement d'un lieu et d'une culture. Le nom d'Hubert Aquin - membre du comité de direction de Liberté de 1961 à 1971 - n'apparaît pas dans «Assoiffés de sens ». Néanmoins, les multiples références au devoir de résistance de l'intellectuel à la répression contemporaine, non

31 Yvon Rivard, "L'héritage de la pauvreté», Personne n'est une île, Montréal, Boréal, coll. "Papiers collés», 2006, p. 134. 32 Le dossier «La résistance culturelle» de la revue Liberté (vol. XLVIII, n 3, septembre 2006) marque l'arrivée de Robert Richard au comité de rédaction et le départ de Marc André Brouillette. 33 Le comité de rédaction, "Assoiffés de sens», Liberté, vol. XLVIII, n³, septembre 2006, p. 3. 
plus liée à la censure cléricale, mais plutôt au nouvel ordre économique, rappellent le propos de certains essais qu'Aquin a justement fait paraître dans les pages de Liberté. Cette réactualisation des idées d'Aquin, certes spectrale dans "Assoiffés de sens", se fera cependant de façon explicite dans plusieurs des articles publiés entre 2006 et 2012 par certains membres du comité de rédaction de la revue, Pierre Lefebvre et Robert Richard en tête.

Il faut noter d'emblée que ces lecteurs ne s'intéressent que très peu au mythe et à la thanatographie de l'auteur, mais bien davantage à ses œuvres romanesques et essayistiques, et plus particulièrement aux rapports entre les idées défendues dans les œuvres et l'état de la culture contemporaine ${ }^{34}$. En témoignent notamment les textes parus à la suite du «Combat des livres» de 2006, qui insistent tous sur la puissance d'évocation et sur les qualités littéraires de l'œuvre aquinienne. Mieux, le congédiement de Prochain épisode par les invités du «Combat des livres» serait le symptôme d'un appauvrissement culturel généralisé, voire d'une quasi-éclipse de la pensée littéraire dans l'espace public. Le rejet de Prochain épisode équivaudrait donc à un «refus de la modernité», mais plus encore de la «séparation des mots et des choses ${ }^{35}$ » chez Olivier Kemeid, à un rejet de «ce qui est de l'ordre de l'exception, de l'anomalie comme de l'exceptionnel, bref de la littérature ${ }^{36}$ » chez Pierre Lefebvre, et à un véritable «boulot de mise à l'index [...] pris en charge par des people (entendez: des personnalités médiatiques) dont l'influence sur nos pratiques collectives de lecture est d'une efficacité à faire crever d'envie le plus exalté des Savonarole ${ }^{37}$ » chez Robert Richard.

Plusieurs aspects importants ressortent de la lecture des articles parus autour du «Combat des livres». D'emblée, les auteurs présentent un sombre portrait de la culture et de la conscience contemporaines, lesquelles accueilleraient plus difficilement l'abstraction, le décentrement et la distance que cultiverait la littérature. Assoiffé non pas de sens, mais de connivence et d'effets de réel, le lecteur contemporain conférerait aux œuvres littéraires un rôle éthique, thérapeutique, utile, et souhaiterait retrouver en un roman de fidèles reflets de lui-même. Consensuelle et bon-ententiste, l'institution littéraire en aurait contre la déviance et l'exceptionnel - emblématisés entre autres par l'œuvre d'Aquin - et privilégierait désormais des écritures prévisibles et peu exigeantes. Une telle subversion de l'acte de lecture ne serait pas uniquement le lot des lecteurs du dimanche, mais affecterait aussi dans une moindre mesure la critique littéraire et le milieu universitaire, trop soucieux de respecter une version expurgée de l'histoire littéraire québécoise. Dans son article «Scouiner la littérature nationale pour lire Aquin... », Robert Richard s'en prend aux canons de l'histoire littéraire du Québec, à ces Maria Chapdelaine qui ont «creusé le chenal étroit que toute œuvre québécoise de bonne famille se doit d'emprunter, dans son long périple vers la

$$
++
$$

34 Dans son article «Ceci n'est pas un Québécois», Pierre Lefebvre s'en prend ouvertement au mythe d'Aquin: «De son côté, Aquin, qu'on ne lit plus trop, n'avait de toute façon à opposer à la machine d'État, à partir de 1977, que ce mythe d'écrivain maudit qu'on n'arrêtait pas de lui coller au sépulcre, lui d'ailleurs qui, c'est le comble, souhaitait voir mourir cette appellation.» Pierre Lefebvre, "Ceci n'est pas un Québécois», Liberté, vol. L, n 1, février 2008, p. 68-69. 35 Olivier Kemeid, «Le refus de la modernité», p. 88. 36 Pierre Lefebvre, «Entre le signe et les choses», Liberté, vol. XLVIII, n 4, novembre 2006, p. 7. 37 Robert Richard, «Scouiner la littérature nationale pour lire Aquin... », Liberté, vol. XLIX, n 4, novembre 2007, p. 70. 
Révélation Nationale ${ }^{38}$ ». Au même titre que La Scouine d'Albert Laberge, l'œuvre d'Aquin, d'ailleurs qualifiée de "déviante» par Pierre Lefebvre ${ }^{39}$, ne serait pas sortable ${ }^{40}$, porterait en elle une langue, un style et une pensée qui « refuseraient d'ériger ou de construire quoi que ce soit ${ }^{41}$ »; mieux, qui «procéde[raient] à une inversion des signes propres au dispositif identificatoire ${ }^{42}$ ».

Il importe toutefois de noter que cette exigence de la littérature n'est pas assimilée à une forme d'élitisme. Dans «Le refus de la modernité», Olivier Kemeid insiste sur le mépris qu'affichent les people du «Combat des livres» envers les lecteurs québécois :

\begin{abstract}
Je n'ai pas et n'aurai jamais un mépris des Québécois au point de penser qu'ils n'ont pas la capacité de lire leurs plus grands écrivains : pour avancer une telle affirmation, dieux du ciel, faut-il ressentir à ce point un complexe d'infériorité! Faut-il prendre sa propre nation et son propre peuple pour un vaste ensemble de gagne-petit, d'ignares et de tarés? [...] À tous ceux qui ont eu la chance d'apprendre à lire, qui peuvent se doter d'un dictionnaire et d'une confiance - c'est cette confiance que nous nous devons de donner aux gens, il est là le rôle des intellectuels, alors aux armes $^{43}$ !
\end{abstract}

Le même type de constat apparaît sous la plume de Pierre Lefebvre:

Je ne voudrais pas non plus que l'on puisse croire [...] que je considère la poutine, Passe-Partout ou Juste pour rire indignes de participer à notre distinction, mais bien que celle-ci ne saurait, et ne devrait, se résumer à ce type de manifestations moins complexes à créer et à s'approprier que, par exemple, Arbres de Paul-Marie Lapointe ou Raisons communes de Fernand Dumont. La distinction hermétique entre arts majeurs et arts mineurs m'est toujours apparue suspecte et la vitalité d'une culture consiste en grande partie dans le dialogue fécond entre ses œuvres dites populaires et ses dites grandes œuvres, mais aujourd'hui, au Québec, ce dialogue a disparu car la règle, chaque fois, ordonne plus ou moins gentiment à l'exception de se fermer la gueule ${ }^{44}$.

Ces deux passages sont d'une importance capitale, car ils mettent en évidence certaines des critiques les plus souvent adressées par Liberté à la culture québécoise contemporaine, soit son nivellement par le bas, son désir d'abolir toute forme de hiérarchie et, surtout, son mépris de l'exception. Or en feignant de démocratiser la littérature, de la rendre accessible au plus grand nombre, les membres de l'industrie culturelle ne parviendraient qu'à imposer une forme de totalitarisme pervers, d'uniformisation de la pensée qui conduirait à l'élimination de la complexité, du raffinement esthétique et de la subversion propres aux œuvres majeures. Le dialogue entre œuvres mineures et œuvres majeures aurait disparu dans le Québec contemporain, tant et si

38 Ibid., p. 71.39 Pierre Lefebvre, «Entre le signe et les choses», p. 6. 40 Voir Robert Richard, «Scouiner la littérature nationale pour lire Aquin...», p. 72. 41 Ibid., p. 75.42 Ibid., p. 77. 43 Olivier Kemeid, «Le refus de la modernité », p. 85.44 Pierre Lefebvre, «Entre le signe et les choses», p. 10. 
bien que les amateurs de culture n'auraient plus la chance de choisir, de discriminer, d'élire et, surtout, de s'approprier des textes exigeants.

Et l'œuvre d'Aquin dans tout cela, ne serait-elle qu'un simple prétexte? Oui et non. Oui, car elle sert de bouclier contre les assauts de la culture médiatisée. Elle permet ainsi aux auteurs d'ouvrir leurs réflexions à des problématiques plus amples, inscrites à la fois dans les sphères culturelle et politique, ce qui fait directement écho au manifeste "Assoiffés de sens ». Le nouveau comité de rédaction de Liberté s'y refusait en effet au cloisonnement des discours et manifestait le désir de faire de la littérature "un lieu de parole ${ }^{45}$ ». Non, car elle est chaque fois véritablement lue, ne serait-ce que de manière allusive. "La fatigue culturelle du Canada français », Prochain épisode et Neige noire, les trois textes aquiniens les plus souvent convoqués par Lefebvre et Richard, ne sont considérés comme exemplaires que dans la mesure où ils résistent à toute lecture univoque et purement utilitaire. $\mathrm{Si}$, comme le note Robert Richard, ce passage : « [... . il n'y a plus de nation canadienne-française, mais un groupe culturellinguistique homogène par la langue ${ }^{46}$ ", tiré de «La fatigue culturelle du Canada français», a pu inspirer à certains lecteurs contemporains des interprétations identificatoires sur les bienfaits du multiculturalisme, les œuvres publiées par Aquin après 1965 s'attacheraient à «ce qui est extérieur ou étranger à la langue commune et, finalement, à la nation ${ }^{47}$ ». La langue aquinienne, langue étrangère, illustrerait éloquemment ce désir de mise à distance des mots de la tribu.

Dans les articles qui paraissent ultérieurement, et qui ne mentionnent pas le «Combat des livres", l'œuvre d'Aquin est tour à tour convoquée pour éclairer la situation politique québécoise contemporaine, l'histoire de la littérature du Québec et le rapport au religieux dans une société laïque. Dans «Le péché de Westphalie» de Robert Richard et «Ceci n'est pas un Québécois» de Pierre Lefebvre, parus respectivement en 2006 et en 2008, Aquin est relu de manière à mettre à distance - encore une fois - l'insidieuse influence de la nation sur la sphère culturelle et la conception de la littérature québécoise. Chez Richard, le péché de Westphalie, hérité des fameux traités de 1648 conférant des frontières inviolables aux états européens, consisterait à concevoir les littératures à l'aune d'obsessions nationales. Loin d'avoir disparu à l'époque contemporaine, il subvertirait les propos de nombreux écrivains québécois, Jacques Godbout et Madeleine Gagnon en tête. À l'instar de Nietzsche, d'Adorno, de Celan et de Szondi, Aquin «laver[ait] ce monde du péché de Westphalie ${ }^{48}$ » :

\footnotetext{
Aquin - l'écrivain Aquin - a mis le cap sur le Verbe... Et c'est le défi qu'il lance à la société québécoise, mais pas seulement à cette société. Car la question est bien de savoir ce que l'écrivain doit faire dans ce monde tout encombré de drapeaux? La réponse : poursuivre son périple en haute mer vers le Verbe ou vers l'Être, pourraiton dire en s'inspirant de Heidegger ${ }^{49}$.
}

45 Le comité de rédaction, "Assoiffés de sens», p. 4. 46 Guy Scarpetta et Robert Richard, "Dialogue sur Neige noire d'Hubert Aquin", Liberté, vol. LI, n 3, février 2010, p. 120. 47 Robert Richard, «Scouiner la littérature nationale pour lire Aquin... », p. 79.48 Robert Richard, "Le péché de Westphalie», Liberté, vol. XLVIII, n 4, novembre 2006, p. 88. 49 Guy Scarpetta et Robert Richard, «Dialogue sur Neige noire d'Hubert Aquin », p. 107. 
La lecture de Pierre Lefebvre, quant à elle, rejoint obliquement les vues de Robert Richard. N'allant pas jusqu'à dépolitiser «La fatigue culturelle du Canada français», Lefebvre se demande pourquoi les constats de Trudeau sur le bilinguisme et le multiculturalisme ont eu droit de cité alors que les propositions d'Aquin ont été oubliées:

\begin{abstract}
[Aquin] se demande en effet, entre autres : pourquoi est-ce que je devrais, pour justifier mon existence collective, me mettre à briller dans le regard de l'autre? Pourquoi à une problématique politique, donc collective, me faudrait-il répondre en tant qu'individu? Il semble d'ailleurs que le temps lui ait donné raison: Maurice Richard, les trois colombes du «French Power", le prix cannois de la mise en scène de Michel Brault pour Les ordres, Céline Dion, le Cirque du Soleil, l'Ours d'or de Martine Chartrand pour Âme noire, le prix Europe de Robert Lepage, les prix d'interprétation féminine, à Cannes encore, de Marie-Josée Croze et de Monique Mercure, en passant par Julie Payette et Marc Garneau dans l'espace, l'Oscar de Denys Arcand ou les succès internationaux de Wajdi Mouawad et de Michel Tremblay, de même que les wagons de métro bien de chez nous à Chicago ou à Paris, n'ont en rien, que je sache, ralenti, encore moins empêché, le taux d'assimilation galopante des communautés francophones hors Québec ${ }^{50}$.
\end{abstract}

La fidélité à l'œuvre d'Aquin se joue ici doublement. D'une part, Richard et Lefebvre sont tous deux conscients des limites d'une pensée de l'engagement littéraire fondée sur une trop stricte homologie entre littérature, voire culture, et politique. Même s'ils entendent faire de la littérature un «lieu de parole », ils ne souhaitent pas pour autant renouer avec une lecture trop étroitement sociologique du fait littéraire, bien au contraire. La littérature constituerait plutôt, au même titre que les autres discours, un point de vue valable sur le monde, une manière de comprendre les enjeux sociaux et politiques qui animent l'espace public. Cette volonté se traduit, d'autre part, par un respect du caractère équivoque de l'œuvre d'Aquin. Si cette dernière résiste aux rapatriements, c'est bien parce qu'elle ne saurait choisir son camp, hésitant à privilégier de manière franche l'esthétisation du politique ou la politisation de l'esthétique ${ }^{51}$, comme en font foi les romans et les essais publiés par Aquin à partir de 1965.

La question du rapport au religieux, aussi traitée par Jacques Beaudry dans ses deux essais, affleure à quelques reprises chez Robert Richard, et plus particulièrement dans son entretien avec Guy Scarpetta ${ }^{52}$. Au tout début de l'entretien, Robert Richard rappelle les circonstances ayant entouré sa soutenance de thèse en avril 1984, «houleuse » précisera-t-il. Dans sa thèse, intitulée La voix de l'inceste dans l'œuvre d'Hubert Aquin, il montrait que "la référence à la Bible et au catholicisme était soutenue et pire encore qu'elle faisait système, dans l'œuvre romanesque de la maturité ${ }^{53}$ ». Sa

$$
++
$$

50 Pierre Lefebvre, «Ceci n'est pas un Québécois», p. 67. 51 Voir à ce sujet les pages que Martin Jalbert consacre aux «révolutions contrariées» d'Aquin dans Le sursis littéraire. Politique de Gauvreau, Miron, Aquin, Montréal, Presses de l'Université de Montréal, coll. «Nouvelles études québécoises», 2011, p. 151-188. 52 À ce sujet, voir aussi «L'Europe en Amérique», chapitre consacré « au sens anagogique du texte aquinien », Robert Richard, L'émotion européenne: Dante, Sade, Aquin, Montréal, Varia, coll. «Philosophie», 2004, p. 161. 53 Guy Scarpetta et Robert Richard, «Dialogue sur Neige noire d'Hubert Aquin», p. 107; les auteurs soulignent. 
thèse fut contestée par certains membres du jury car elle ne convenait pas à l'esprit de l'époque:

\begin{abstract}
ceux qui m'écoutaient - mes juges, d'une certaine façon - avaient été, c'était le cas de beaucoup d'entre eux, parmi les artisans de la Révolution tranquille au Québec. Il leur était donc intolérable qu'un blanc-bec comme moi vienne leur dire qu'un des hérauts de leur Québec moderne et laïque avait produit une œuvre saturée de références au catholicisme ${ }^{54}$
\end{abstract}

L'anecdote peut sembler bien anodine. Pourtant, elle illustre éloquemment les rapports qu'entretiennent les auteurs de Liberté avec l'œuvre d'Aquin. Cette dernière s'inscrit, on l'a souvent dit, en marge des mots d'ordre et des discours officiels, mais plus encore elle pense ce monde-ci à contretemps, avec une sorte de distance volontairement entretenue qui redonne à la littérature la faculté de multiplier les chambardements et les perturbations. Chez les auteurs de la revue Liberté, Aquin est l'anachronique écrivain qui, par-delà le temps et les différentes mouvances idéologiques, survit non seulement à son époque, mais aussi à celles qui lui ont succédé. Il serait ainsi - et fort paradoxalement - celui qui pointerait la mort d'une certaine idée de la littérature - exigeante, engagée, insoucieuse des pouvoirs politiques et économiques - et la potentielle survie de cette même idée à l'époque contemporaine. Il serait une sorte de symptôme, au sens où l'entend Georges Didi-Huberman: « un symptôme ne survient jamais au bon moment, apparaît toujours à contretemps, tel un très ancien malaise qui revient importuner notre présent ${ }^{55}$ ».

Une dernière remarque s'impose. Il serait trop simple d'en conclure que l'œuvre d'Aquin est, pour les auteurs de Liberté, l'emblème d'une Révolution tranquille figée dans le temps, dont les leçons seraient ponctuellement réactualisées. Si la période de la Révolution tranquille demeure un moment charnière dans l'histoire de la revue et par là même une référence dans l'imaginaire du comité de rédaction, le grand récit qui l'accompagne est fort souvent égratigné, remis en cause, interrogé. La Révolution tranquille fut également l'occasion d'entretenir un leurre sur l'identité collective du Québec moderne : «le label québécois est [...] soudainement apparu comme un garant de sa modernité et de sa maturité, lui évitant du même coup de s'astreindre au travail nécessaire pour véritablement acquérir ces dernières ${ }^{56}$ ». Le désenchantement contemporain, l'absence de débats, le calme politique, la professionnalisation de la littérature seraient ainsi logiquement des héritages de la Révolution tranquille. Parce qu'il a disparu avant la "dégringolade ${ }^{57}$ ", pour reprendre le mot de Robert Richard, parce que son œuvre aurait été en marge des discours lyriques sur la nation québécoise, Aquin n'a pu souscrire à cette postérité-là.

54 Ibid., p. 107-108. 55 Georges Didi-Huberman, Devant le temps. Histoire de l'art et anachronisme des images, Paris, Éditions de Minuit, coll. «Critique», 2000, p. 40. 56 Pierre Lefebvre, "Entre le signe et les choses», p. 12. 57 Robert Richard, «Le péché de Westphalie», p. 79. 
Mais quelle est alors la postérité d'Aquin ? Quels héritages a-t-il légués aux contemporains? Une parole mythique et sacrificielle, une thanatographie vouant l'Histoire de la communauté à l'impasse ou une leçon d'engagement littéraire? Difficile de répondre à une telle question en respectant les singularités des lectures et des points de vue. Néanmoins l'analyse tend à montrer qu'à l'œuvre et à la figure d'Aquin est conféré un caractère d'exception, c'est-à-dire des qualités d'analyse et d'écriture comparables à celles des grands auteurs européens, mais surtout une mémoire de la littérature québécoise. Et c'est par ce biais que le mythe d'Aquin, avec toutes les précautions méthodologiques que suppose l'emploi d'un tel syntagme, communique et communie avec ses lecteurs. Catherine Mavrikakis, dans ses essais comme dans son roman Ça va aller, a bien montré comment le legs aquinien témoignait in absentia d'un autre devenir - devenir potentiel, s'entend - de la littérature québécoise. Selon elle, Aquin «s'inscrit déjà dans une pensée d'une possibilité d'une fin de l'histoire moderne, de répétition qui annule le déploiement du temps et dont le minoritaire serait porteur ${ }^{58}$ ». Comparable aux dérapages, aux improvisations et aux explosions mis en scène dans les textes aquiniens, ce détraquage temporel aurait pour effet de ralentir la marche moderne vers le progrès, d'en "paralyser l'évolution ${ }^{59}$ ». Or les lectures contemporaines d'Aquin vont presque toutes dans ce sens. Qu'elles tendent à exacerber le sacrifice de l'écrivain, à recréer une communauté de suicidés de la société ou à repenser les textes aquiniens dans le contexte d'une crise de la culture et de la transmission, elles disent encore une fois la valeur anachronique d'une œuvre qui ne tolérerait pas les demi-mesures. On pourrait bien sûr reprocher aux lecteurs contemporains d'Aquin de procéder à une forme d'héroïsation parfois suspecte de l'écrivain - à laquelle s'en prend d'ailleurs Richard Dubois dans son Hubert Aquin blues. Mais ce serait dépouiller le travail de la lecture de son affectivité et lui dénier sa part existentielle. Héros sans doute, mais héros bien imparfait, Aquin est, pour Jacques Beaudry, Guylaine Massoutre et Yvon Rivard comme pour les auteurs de la revue Liberté, une sorte de survivant. Il est l'un des derniers témoins, non d'une époque clairement circonscrite, mais d'une certaine conception de la littérature vue comme un art exigeant, un travail formel, une parole complexe alliant des réflexions politiques et littéraires. L'œuvre d'Aquin, actualisée par ces lecteurs qui auront prononcé «le verdict qui décidera de [son] sérieux et de [son] authenticité» $(M 2,348)$, tiendrait ainsi à la fois du propre et de l'étranger, de l'inédit et de la répétition, du passé et du présent.

58 Catherine Mavrikakis, « "Qu'on en finisse donc..." : l'inscription du posthume, de la survivance et du prénatal modernes », Ginette Michaud et Élisabeth Nardout-Lafarge (dir.), Constructions de la modernité au Québec. Actes du colloque tenu à Montréal les 6, 7 et 8 novembre 2003, Outremont, Lanctôt éditeur, 2004, p. 312.59 Ibid., p. 313. 